\title{
Neuroglycopenia Presented as Acute Cerebellar Stroke on Neuroimaging : A Rare Case Report
}

\author{
Ibraheem Khan ${ }^{1 *}$, Yadvendra Gupta ${ }^{2}$ \\ ${ }^{1 *}$ Consultant Neurology, Department of Neurology, Manipal Hospital Jaipur, Rajasthan, India. \\ ${ }^{2}$ Senior Resident Department of Neurology, Manipal Hospital Jaipur , Rajasthan, India. \\ imedicine2008@gmail.com
}

*Corresponding Author: Dr. Ibraheem Khan, VPO-Samraya, weir, Dist-Bharatpur(Raj), India.

\section{Abstract}

Neuroglycopenia commonly occurs in diabetics patients.It manifests as aphasia, paresis, ataxia, seizure, confusions and coma. Here we are presenting a 65 yrs old male patient presented as transient hemiparesis and gait ataxia. There was diffusion restriction in Right cerebellum attributing to low blood sugar level $37 \mathrm{mg} / \mathrm{dl}$. Hypoglycemia can be present with any neurological manifestations however diffusion restriction in cerebellar region is a rarely detected.

\section{INTRODUCTION}

The brain requires continuous supply of glucose for proper functioning . Human brain accounts for $2 \%$ of body weight but it utilises only one fourth of body's glucose due to its high metabolic rate [1]. Uptake of glucose in most cells ( except liver and brain) is dependent on insulin and mediated through various glucose transporters or sodium glucose co-transporter. Neuronal uptake of glucose is non insulin dependent and through glucose transporter[2].

Hypoglycemia commonly occurs in diabetic patients who are receiving either insulin or long acting sulfonylureasand patients with insulin-secreting tumors, sepsis, Addison disease, and hepatic or renal failure[3]. Neuroglycopenia may present with any neurological manifestations including aphasia, paresis, ataxia, chorea, Seizure or coma[4]. Rarely neuroglycopenia can present as ataxia after a hypogly cemicepisode[5].

The Brain areas most vulnerable to hemodynamic alterations such hypoxia, hypotension and hypoglycaemia are subiculum, caudate nucleus, hippocampus, dentate gyrus and superficial cortical layers 2 and 3[6]. MR imaging is the investigation of choice in acute hypoglycaemia setting to determine diagnosis and prognosis. Hypoglycaemia is usually mimic stroke clinically as well as radiologically[7].

Here we are reporting a case of 65 years old male diabetic presented with transient hemiparesis, gait ataxia and acute DWI restriction in Rt cerebellar region( acute cerebellar stroke).

\section{CASE REPORT}

73 year old male patient, presented with complaints of sudden onset-drowsiness, slurring of speech. He has past history of DM type2, CAD-Post CABG, HTN, COAD.

General Physical Examination was unremarkable and his Vital parameter wereblood pressure $-170 / 67 \mathrm{~mm}$ $\mathrm{Hg}$, Pulse-88/min and respiratory rate-26/min.

Neurological examination revealed drowsiness dysarthria, pupils b/l reactive, Motor-moving all 4 limbs spontaneously, normal reflexes and mute bilateral plantar. Sensory and cerebellar signs could not be examined.His routine biochemistry was normal except hemoglobin (Hb-9.2 gm/dl). His blood sugar and $\mathrm{HbA} 1 \mathrm{c}$ were $37 \mathrm{mg} / \mathrm{dl}$ and $5.8 \%$ respectively. So he is diagnosed as hypoglycemic encephalopathy was made and $25 \%$ glucose intravenously was given. After glucose infusion patient regained consciousness and 
Neuroglycopenia Presented as Acute Cerebellar Stroke on Neuroimaging : A Rare Case Report

followed verbal command. After sometime patient was mobilised from bed then we noticed that he developed walking difficulty in form of swaying either side. His other cerebellar signs were normal except gait ataxia. MRI brain with DWI

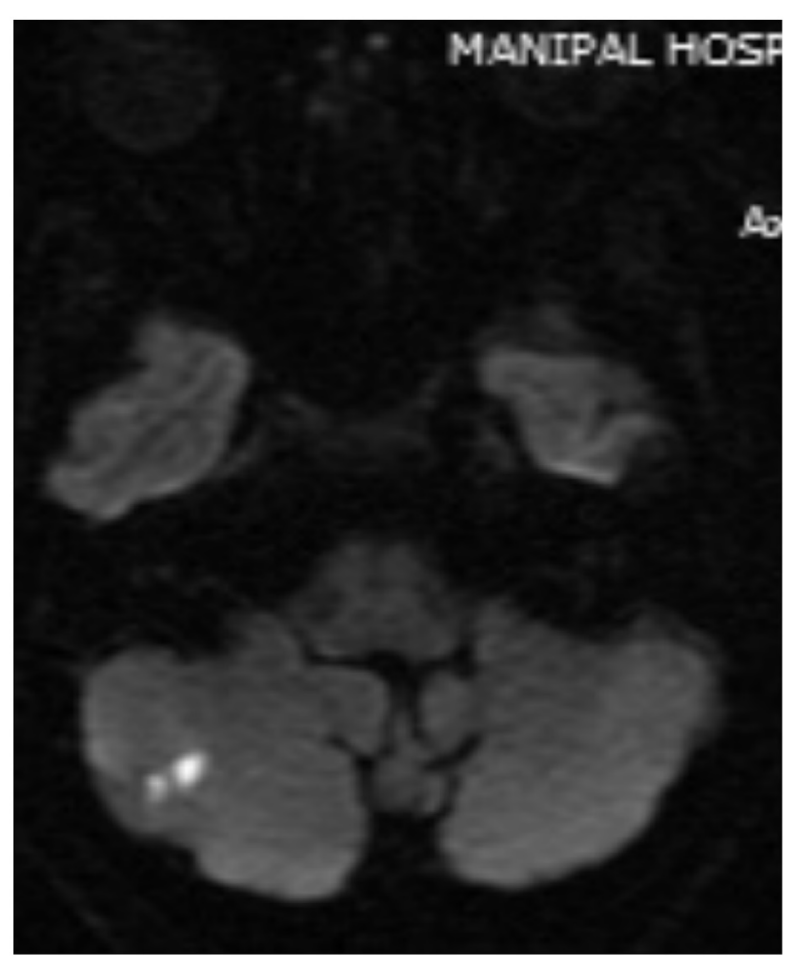

was conducted. MRI brain revealed acute lacunar infarcts in right cerebellar hemisphere[Figure]. Ataxic gait improved after IV Dextrose therapy before $24 \mathrm{hr}$ and was discharged without any focal neurological deficits.

Figure. MRI Brain DWI showing diffusion restriction in Right cerebellar region and corresponding low ADC value.

\section{DISCUSSION}

Hypoglycemic manifestation categories into autonomic, which include sweating, trembling, palpitations, and anxiety and neuroglycopenic, which include weakness, confusion, personality changes, seizures, dyskinesia, headache and transient cognitive impairment[8]. The energy failure due to aspartate neurotoxin in hypoglycemicinduced brain damage leading to loss of cellular homeostasis is well known hypothesis demonstrated in various literature in contrast to ischemic stroke in which cellular damage secondary to glutamate[9]. Aspartate causes selective neuronal damage in the cerebral, basalganglia and hippocampus. This effect is more vulnerable in acute phase result in hyperkinetic movement disorder[10].

Recent case report have shown ADC and DWI changes in hypoglycaemic patients. These changes usually reverse after euglycemia unlike ischemic stroke.

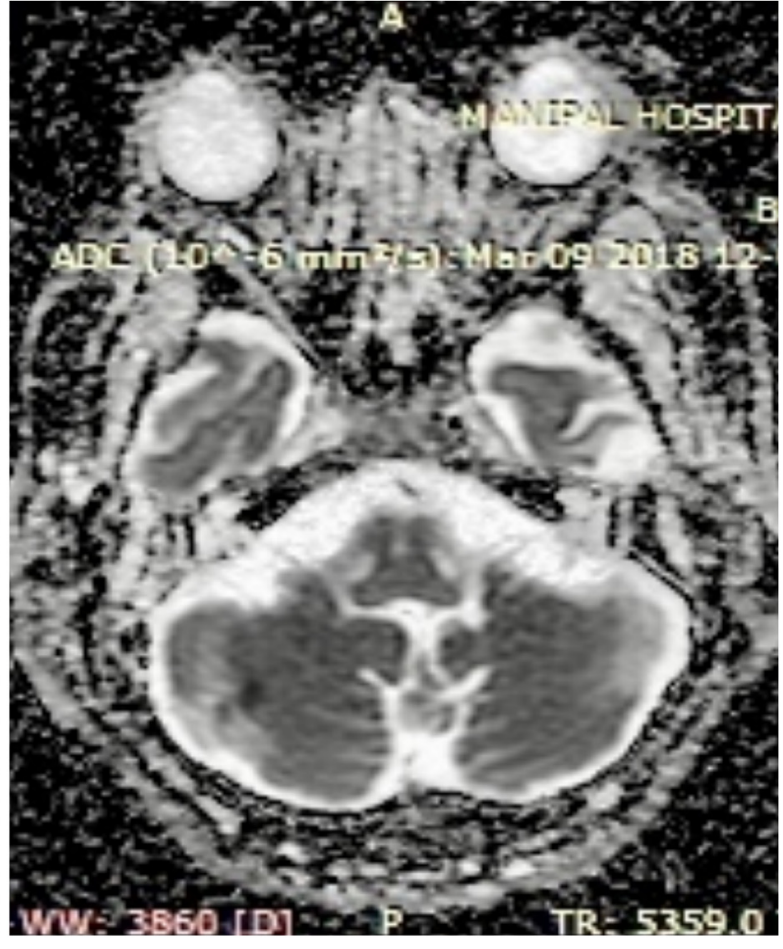

Hypoglycaemic changes are usually bilateral involving mainly basal ganglia region and cortex[9,10].

Thalamus, brainstem and cerebellum are usually not involved in hypoglycaemia. If involved lesions are usually bilateral and unilateral lesion rarely reported. Thalamus is not involved in hypoglycaemia contrast to ischemia [11]. Recently cerebellar ataxia due to hypoglycemia was reported in absence of diffusion restriction on MRI[12].

\section{Conclusion}

Hypoglycemiccan present as any neurological manifestations. The cerebellar ataxia due to hypoglycaemia is rare. The diffusion restriction in unilateral cerebellar region is also very rare. Possibility of hypoglycemia should be considered in all cerebellar stroke patients. Prompt glucose infusion in hypoglycaemic patients completely resolve the signs and symptoms and further prevent brain neurotoxicity. 
Neuroglycopenia Presented as Acute Cerebellar Stroke on Neuroimaging : A Rare Case Report

\section{REFERENCES}

[1] Cryer PE, Davis SN. Hypoglycemia - In: Longo DL, Fauci AS, Kasper DL, et al, eds. Harrison's Principles of Internal Medicine. 18th ed. New York: McGraw-Hill Co; 2012:3003-09.

[2] Hall JE. Guyton and Hall Textbook of Medical Physiology. Philadelphia: Saunders Elsevier; 2011: 809-18.

[3] Burns CM, Rutherford MA, BoardmanJP, et al. Patterns of cerebral injury and neuro develop mental outcomes after symptomatic neonatal hypoglycemia. Pediatrics 2008;122:65-74.

[4] Jagota P, Bhidayasiri R, Lang AE. Movement disorders in patients with diabetes mellitus. J Neurol Sci 2012;314:5-11.

[5] Berz JP1, Orlander JD. Prolonged cerebellar ataxia: An unusual complication of hypoglycemia. J Gen Intern Med 2008;23:103-5.

[6] Kang EG, Jeon SJ, Choi SS, et al - Diffusion MR imaging of hypoglycemic encephalopathy. AJNR Am J Neuroradiol 2010; 31:559-64.
[7] G. Bathla, B. Policeni, and A. Agarwal Neuroimaging in Patients with Abnormal Blood Glucose Levels. AJNR 2013r. A3486.

[8] Yoshino T, Meguro S, Soeda Y, et al. A case of hypoglycemic hemiparesis and literature review. Ups J Med Sci 2012;117:347-51.

[9] Ma JH, Kim YJ, Yoo WJ, et al. MR imaging of hypoglycemicencephalopathy:lesion distribution and prognosis prediction by diffusion-weighted imaging. Neuroradiology 2009; 51: 641-49.

[10] Schmidt P, Bo"ttcher J, Ragoschke-Schumm A, et al. Diffusion weighted imaging in hyperacutecerebral hypoglycemia. AJNRAmJ Neuroradiol 2011;32:1321-27.

[11] FujiokaM, OkuchiK, HiramatsuK, et al. Specific changes in human brain after hypoglycemic injury. Stroke 1997;28:584-87.

[12] Agrawal N, Jamshed N, Aggarwal P, Ekka M. Severe Hypoglycemia Masquerading as Cerebellar Stroke. J Family Med Prim Care 2014; $3: 440-442$.

Citation: Ibraheem Khan, Yadvendra Gupta. Neuroglycopenia Presented as Acute Cerebellar Stroke on Neuroimaging : A Rare Case Report. Archives of Emergency Medicine and Intensive Care. 2018; 1(2): 27-29.

Copyright: (C) 2018 Ibraheem Khan, Yadvendra Gupta. This is an open access article distributed under the Creative Commons Attribution License, which permits unrestricted use, distribution, and reproduction in any medium, provided the original work is properly cited. 